\title{
Zarzuela: Spanisches Musiktheater - ein Projekt am Ibero-Amerikanischen Institut
}

\author{
Ulrike MÜHLSCHLEGEL (Berlin)
}

Die Bibliothek des Ibero-Amerikanischen Instituts (IAI) in Berlin besitzt mit über 1.600 Exemplaren eine umfangreiche Sammlung von spanischen und hispanoamerikanischen Zarzuelas aus dem 19. und 20. Jahrhundert. Der Schwerpunkt liegt auf Werken, die in Spanien zwischen 1800 und 1930 gedruckt wurden. Der Gattungsbegriff Zarzuela umfasst dabei auch Formen wie Entremeses und Sainetes, deren Entwicklung ab ca. 1900 mit der Zarzuela weitestgehend zusammenfiel. Zarzuela-Libretti wurden vor allem im 19. Jahrhundert aus Kostengründen auf dünnem, fragilem Papier gedruckt. Sie sollten die populären Texte preiswert weiter verbreiten und die Zarzuela-Aufführungen begleiten. Aufgrund dieser mangelnden Qualität der Druckerzeugnisse sowie ihres populären Charakters sind Zarzuela-Texte in zeitgenössischen Bibliotheken nur selten erhalten. Die Sammlung des IAI ist aufgrund ihrer Größe und ihres Inhaltes in Deutschland einzigartig, die große ehemalige Sammlung der Staatsbibliothek zu Berlin ging im 2. Weltkrieg verloren.

Galt die Zarzuela in der zweiten Hälfte des 20. Jahrhunderts als nationalistisch vereinnahmte und eng mit der spanischen Franco-Diktatur verbundene Gattung, ist seit der Jahrtausendwende ein erneuter Aufschwung in der Aufführungspraxis in spanischen wie auch allgemein in europäischen Theatern festzustellen. So feierte z.B. in der Saison 2016/2017 mit Luisa Fernanda im Landestheater Nordhausen erstmals wieder eine deutschsprachige Produktion Premiere. Auch das wissenschaftliche Interesse wächst deutlich (cf. z.B. die Tagungen und Tagungsbände von Brandenberger 2014 und Brandenberger/Dreyer 2016) ${ }^{1}$. Dabei stellt die Verfügbarkeit der älteren Originaltexte, die aufgrund ihres schlechten Erhaltungszustandes in den Bibliotheken und über die internationale Fernleihe oftmals nicht zugänglich sind, ein großes Desiderat für Forscherinnen und Forscher dar.

Mit dem von der Beauftragten der Bundesregierung für Kultur und Medien (BKM) geförderten Projekt werden die Zarzuela-Libretti des IAI durch eine fachgerechte Digitalisierung nach DFG-Richtlinien ortsunabhängig zugänglich und für die Forschung nutzbar gemacht. Über eine OAI-Schnittstelle werden die Daten zum Download bereitgestellt und können so in eigene Forschungsumgebungen eingebunden und nachgenutzt werden. $\mathrm{Zu}$ gleich wird das vom Papierzerfall bedrohte Kulturgut dauerhaft erhalten.

Um das multimediale Genre in seinen Aspekten Bild, Ton und Text darzustellen und der Öffentlichkeit zu vermitteln, wurde vom 8.12.2016 bis 25.2.2017 die Ausstellung Zar- 
zuela: Spanisches Musiktheater im Lesesaal des IAI gezeigt. Dazu ist ein Begleitkatalog ${ }^{2}$ mit Abbildungen und wissenschaftlichen Aufsätzen erschienen, der ebenfalls zum kostenlosen Download zur Verfügung steht.

Nach der Erschließung und der Digitalisierung der Zarzuela-Libretti Ende 2017 stehen ca. 1.600 Titel über den OPAC der Bibliothek ${ }^{3}$ sowie die Digitalen Sammlungen des IAI ${ }^{4}$ zur Verfügung. Die Ausstellung ist als Wanderausstellung konzipiert und kann auf Anfrage nachgenutzt werden.

\section{Endnoten}

1 Tobias Brandenberger (Hg.), Dimensiones y desafios de la zarzuela, Berlin et al., LIT, 2014; Tobias Brandenberger / Antje Dreyer (Hg.), La zarzuela y sus caminos. Del siglo XVII a la actualidad, Berlin et al., LIT, 2016.

2 http://www.iai.spk-berlin.de/publikationen/ausstellungskataloge/zarzuela-spanisches-musiktheater-el-mundo-comedia-es-die-welt-ist-eine-buehne.html (Zugriff 14.06.2017).

3 www.iaicat.de (Zugriff 14.06.2017).

4 www.iaidigital.de (Zugriff 14.06.2017). 